THEORIA, 2013, 79, 309-325

doi:10.1111/theo. 12007

\title{
Absences and Late Preemption
}

by

\author{
OISÍN DEERY
}

University of British Columbia

\begin{abstract}
I focus on token, deterministic causal claims as they feature in causal explanations. Adequately handling absences is difficult for most causal theories, including theories of causal explanation. Yet so is adequately handling cases of late preemption. The best account of absencecausal claims as they appear in causal explanations is Jonathan Schaffer's quaternary, contrastive account. Yet Schaffer's account cannot handle preemption. The account that best handles late preemption is James Woodward's interventionist account. Yet Woodward's account is inadequate when it comes to absences. I propose an account that handles both absences and preemption by transposing Schaffer's account into an interventionist framework.
\end{abstract}

Keywords: causation, absences, preemption, causal modelling

\section{Introduction}

WHEN I SAY "I caused my plant to die by not watering it," I am claiming that something I did not do (water my plant) caused something to happen (my plant's dying). In this explanation of why my plant died, an absence is cited as occupying the role of cause. Absences may also figure in our causal explanations by occupying the role of effect, or as disconnections in a causal chain. For present purposes, I will assume that statements citing absences as causes are sometimes judged to be true, and that absences often feature in our causal explanations of various events and sequences of events. ${ }^{1,2}$

In section 2, I outline Jonathan Schaffer's quaternary, contrastive account of causal claims, which understands causation as a four-place relation, flanked on each side by an actual event and a contrastive set of possible alternative events. I leave aside whether Schaffer's account overcomes the problems associated with taking absences to be genuinely causal, while nonetheless endorsing it as an account of

1 I remain neutral on the issue of whether absences are causes.

2 I take it to be an empirical question whether people actually do take absences to be genuinely causal. See Livengood and Machery (2007) for a recent experimental philosophy study on whether the folk take absence causation to be genuine. One result of this study is that in at least some cases participants did not judge that absences are causal, although they did judge that absences are explanatory. Also relevant here is work by Lombrozo (2010), which suggests that people are more willing to cite absences as causes when events are construed teleologically rather than mechanistically, since when events are construed teleologically people are more sensitive to counterfactual dependence (roughly, difference making) than they are to physical connection in causal explanations. 
causal explanation, which makes sense of the way in which we cite absences as causes. Negative nominals such as "My not watering my plant" denote actual events, while absence-talk sets the contrast to the possible event said to be absent.

But Schaffer's account of causal explanation cannot handle cases of late preemption, since it is a counterfactual account. For example, consider the following situation: event $c_{1}$ causes event $e$, but if it had not then event $c_{2}$ would have caused $e$ instead. Especially in cases where both $c_{1}$ and $c_{2}$ actually occur, but $c_{1}$ causes $e$ slightly more quickly that $c_{2}$ would have, it seems that we cannot get counterfactual dependence of $e$ on $c_{1}$. After all, even if $c_{1}$ had not occurred, $e$ would still have occurred due to $c_{2}$.

This is a general problem for counterfactual theories, and it is not overcome by Schaffer. By contrast, an elegant solution to cases of late preemption is provided by James Woodward's interventionist account of causal explanation. ${ }^{3}$ I outline Woodward's account in section 3, and I illustrate how it successfully handles late preemption. Yet Woodward's account is far from ideal when it comes to handling the way in which we cite absences as causes. Although Woodward wants to count absence-causal claims as sometimes being good explanations, a variant of the problem that Schaffer's account overcomes remains as a difficulty for Woodward. In short, the variables in Woodward's causal models are supposed to pick out actual events in the world. But when variables take their default setting (zero) and represent an absence, it is unclear what exactly we are meant counterfactually to imagine occurring. Another way of putting the point is to say that although Woodward's account is implicitly contrastive, the account is prevented from being explicitly contrastive since it remains unclear what it is that absence-causal claims denote.

In section 4, I propose a revised account of causal explanation that handles both absences and late preemption, by transposing Schaffer's account into a causalmodelling framework. Finally, in section $5 \mathrm{I}$ show how my proposed account overcomes the difficulties faced by both Schaffer and Woodward.

\section{Absences}

Schaffer thinks that citing absences in causal explanations is intuitively acceptable: it just sounds right, he thinks, to say that my not watering my plant was what caused it to die. Schaffer also notes that absences play a role in many paradigmatic causal explanations, such as in instances of disconnection, where absences are cited as intermediaries in a causal chain. For example, when we say that decapitating

3 Some take Woodward's account to be a counterfactual theory, although of a different kind to most other such theories. 
someone caused his or her death, we mean that it does so by preventing oxygenated blood from reaching the brain, which in turn would have prevented death. We also tend to cite absences as causes in our explanations of moral and legal responsibility: we explain why a negligent father is both morally and legally responsible for his child's running onto the road and being killed by citing an omission on the part of the father.

Yet viewing absences as being genuinely causal strikes many philosophers as "metaphysically abhorrent." As David Lewis (2000, pp. 195-196) put it, "Absences are not events. They are not anything: where an absence is, there is nothing relevant there at all. Absences are bogus entities." And even apart from the metaphysics of causation, how can we make sense of the fact that we cite such "bogus entities" in our causal explanations?

When it comes to the metaphysics of causation, first, the difficulty for Schaffer is how to categorize causal relata. The standard view is that the relata are events, their number is two, and they occupy the binary roles of cause and effect. Schaffer is concerned with questions about the number and role of relata and their connection to the causal relation. For him, the category of causal relata is that of either (i) events or (ii) contrast event sets; the number of relata is four; and the roles of the relata are that of cause, effect, and contrastive set of events. So causation is a quaternary, contrastive relation for Schaffer, rather than a binary, comparative one. One advantage of Schaffer's account of the causal relata is that it allows us adequately to handle absence-causal talk. ${ }^{4}$ In this way, it also provides an account of causal explanation, at least in so far as we cite absences in many of our typical causal explanations.

There are three assumptions on which Schaffer relies. First, he assumes that the causal relata we cite are events or sets of events (Schaffer, 2005, p. 298). Second, he assumes that counterfactual dependence is a "decent test" for causal relatedness (Schaffer, 2005, pp. 317-318); i.e., difference-making provides a test for contrastive causal explanation with singleton contrasts: $c$ rather than $c^{*}$ causes $e$ rather than $e^{*}$ iff $O\left(c^{*}\right)>O\left(e^{*}\right) .{ }^{5}$ Schaffer notes that this is Lewis's counterfactual test, adapted for quaternicity. Third, the contrast sets $\mathbf{C}^{*}$ and $\mathbf{E}^{*}$ are fixed by context (Schaffer, 2005, pp. 299, 318-319).

First, then, Schaffer assumes that in causal explanation, the relata we cite are events, or sets of events. ${ }^{6}$ His strategy is as follows: "(i) treat negative nominals as denoting actual events, and (ii) treat absence-talk as tending to set the associated contrast to the possible event said to be absent" (Schaffer, 2005, p. 301). Thus,

\footnotetext{
4 Note, though, that some will be unhappy with contrast sets of events as causal relata.

$5 O\left(c^{*}\right)>O\left(e^{*}\right)$ : If the proposition that $c^{*}$ occurs were true, then the proposition that $e^{*}$ occurs would be true.

6 So, for instance, he denies that we cite facts as causal relata.
} 
"My not watering my plant caused my plant to die" is to be interpreted as "My napping rather than watering my plant caused my plant's dying rather than living." On this strategy, "My not watering my plant" denotes an actual event, by being a way of describing an occurrence not by comparison but by contrast. In explaining why my plant died, we cite my napping (an actual event) rather than my watering the plant (a merely possible event) as a cause of the plant's dying rather than living. The absence description sets the contrast.

In this way, contrastivity turns the counterfactual dependence down the right path: " $c$ rather than $c$ " stresses the dependence on my not watering the plant. On a Lewis-style account, we would have " $c$ rather than $\neg c$," wrongly stressing the dependence on my napping. My napping $c$ rather than watering the plant $c^{*}$ caused my plant to wilt, whereas my napping rather than watching TV did not.

The second assumption on which Schaffer relies is that counterfactual dependence provides a "decent test" for causal relatedness: $c$ rather than $c^{*}$ causes $e$ rather than $e^{*}$ iff $O\left(c^{*}\right)>O\left(e^{*}\right){ }^{7}$ Schaffer concedes that this does not provide an analysis of contrastive causation. To develop a contrastive counterfactual analysis, we must restrict the relata.

For example (Schaffer, 2005, p. 318), $c$ rather than $c^{*}$ causes $e$ rather than $e^{*}$ iff i. $\quad c$ and $e$ are actual distinct events;

ii. $\quad c^{*}$ is a possible event alternative to $c$, and $e^{*}$ is a possible event alternative to $e$; and

iii. $O\left(c^{*}\right)>O\left(e^{*}\right)$.

Schaffer next suggests how we might extend such an analysis to sets of contrasts: ${ }^{8}$

$c$ rather than $\mathbf{C}^{*}$ causes $e$ rather than $\mathbf{E}^{*}$ iff

i. $\quad c$ and $e$ are actual distinct events;

ii. $\quad \mathbf{C}^{*}$ is a set of possible events alternative to $c$, and $\mathbf{E}^{*}$ is a set of possible events alternative to $e$; and

iii. there is a one-one mapping from $\mathbf{C}^{*}$ to $\mathbf{E}^{*}$ by counterfactual entailment: $\left(\forall x \in \mathbf{C}^{*}\right)\left(\exists y \in \mathbf{E}^{*}\right)\left(O(x)>(O(y)) \wedge\left(\forall y \in \mathbf{E}^{*}\right)\left(\exists x \in \mathbf{C}^{*}\right)(O(x)>O(y))\right.$

The third assumption on which Schaffer's account relies is that the contrast sets $\mathbf{C}^{*}$ and $\mathbf{E}^{*}$ are fixed by context (Schaffer, 2005, pp. 299, 318-319). As he puts it:

7 The function of the counterfactual $O\left(c^{*}\right)>O\left(e^{*}\right)$ (If the proposition that $c^{*}$ occurs were true, then the proposition that $e^{*}$ occurs would be true) is to track the Lewisian counterfactual $\neg O(c)>\neg O(e)$ (If the proposition that $c$ does not occur were true, then the proposition that $e$ does not occur would be true); it serves this function by referring to the possible alternative events said to be absent.

8 Schaffer also suggests how his analysis extends to the indeterministic case. I leave this aspect of his account aside. 
[W] hat is being assumed is that the contrasts derive from the structure of the causal inquiry. The causal inquiry determines a three-part structure: (i) the background circumstances, (ii) the causal options: $\{c\} \cup \mathbf{C}^{*}$, and (iii) the effectual options: $\{e\} \cup \mathbf{E}^{*} .9$

Finally, in cases where contrastivity fails to resolve a particular difficulty, the resolution should be pragmatic. Thus, the claim that "The queen's reigning on her throne rather than watering my plant caused my plant to die rather than blossom" can be explained away on the basis that we never supposed that the Queen would water my plant. We resist taking such an unrealistic supposition as a contrast; it is not a relevant alternative. ${ }^{10}$ Schaffer $(2005$, p. 318$)$ thinks that his view is compatible with almost any analysis of causation, and even with resistance to analysis. $^{11}$

\section{Late Preemption}

Now let us consider a case of late preemption (cf. Hall, 2004, p. 235):

\section{Billy and Suzy:}

Suzy and Billy are experts at throwing rocks. They pick up rocks and throw them at a bottle. Billy throws his rock at the same time as Suzy but slightly harder, so that his rock gets there first and shatters the bottle. Both throws are accurate, and so Suzy's would have shattered the bottle if Billy's had not. So the shattering is overdetermined. Yet Billy's throw is an actual cause of the shattering, not Suzy's.

Schaffer's account fails for cases of late preemption. ${ }^{12}$ It should come out true that Billy's throwing his rock rather than rubbing his eye caused the bottle to smash rather than remain intact, despite the fact that had he rubbed his eye the bottle would have smashed due to Suzy's having thrown her rock. Thus, Schaffer's account gets preemption cases wrong. ${ }^{13}$

9 Schaffer (2005, p. 319) adds: "This is the Mackian view, on which 'A causal statement will be the answer to a causal question,' where 'Both causes and effects are seen as differences within a field' (Mackie, 1974, 34-35)."

10 This sort of consideration is important for Woodward.

11 This is important, since Woodward explicitly denies that his interventionist account provides a reductive analysis of causation or that such an analysis is required: "The account I present is not reductive" (2003, p. 20). Moreover, Christopher Hitchcock - who develops a view similar to Woodward's - commits himself to providing a reductive analysis of causation only in the following sense: "If, as Lewis believes, the truth values of counterfactuals supervene upon non-causal facts, then the correctness of a set of structural equations does as well" (2001, p. 284).

12 See Schaffer (2005, n.35, p. 328).

13 Although I do not have space to go into the issue here, cases of late preemption such as "Billy and Suzy" are problematic for many theories of causation, and particularly for counterfactual theories such as Lewis's. 
Woodward's account of causal explanation gets preemption cases right. But before I demonstrate how it does so, let me briefly sketch Woodward's interventionist, causal-modelling framework. Central to Woodward's approach is what Christopher Hitchcock (who develops a similar approach) calls explicitly nonforetracking (ENF) counterfactuals. The idea is this. In the case of standard counterfactuals used in making causal claims, it is required that such counterfactuals not backtrack: we must hold fixed the past up until the time at which the event described by the counterfactual antecedent occurs. So if $c$ causes $e$ (but not vice versa), then a counterfactual of the form "If $e$ had not occurred, then ..." must hold $c$ fixed; otherwise we could incorrectly conclude that $e$ causes $c$ (cf. Hitchcock, 2001, p. 275). It is also standardly required that such counterfactuals foretrack: if $c$ causes $e$, then we must not hold fixed event $e$ when evaluating counterfactuals of the form, "If $c$ had not occurred, then ...," since doing so would mean that the consequent of that conditional could not possibly be "e would not have occurred" (cf. Hitchcock, 2001, p. 275). Yet as Hitchcock points out, such a restriction on what must tacitly be held fixed in evaluating counterfactual antecedents does not restrict the antecedents that we are permitted to entertain. The requirement that we foretrack from $c$ to $e$ does not prevent us from entertaining a counterfactual of the form, "If $c$ had not occurred, but e had occurred anyway, then. . ." Hitchcock calls such counterfactuals explicitly non-foretracking. Lewisian counterfactual analyses do not employ ENF counterfactuals, but counterfactuals with simple antecedents requiring us to make stipulations about only one event, whereas ENF counterfactuals require that we make stipulations about two (or more) events.

Here is Woodward's account. First, we need the notion of a causal model, which is a representation that encodes counterfactual relationships between variables. The variables represent the causal relata. The main restriction on what counts as a variable is that it must represent particular events in such a way that they can be set to different values by interventions (cf. Woodward, 2003, pp. 11-14). An intervention is an exogenous change to the value of a variable. ${ }^{14}$ (Endogenous changes occur due to changes in the values of other variables in the model.) Interventions are also "surgical," in the sense outlined in relation to ENF counterfactuals: the usual causes of a variable, or of a variable's taking a given value, are suspended. Finally, the counterfactuals specifying the relations that hold between variables in a model are stated as structural equations, which are asymmetrical: the values of the variables on the left hand side of the "=" are determined by what appears on the right hand side.

The definition of token causation requires the notion of a direct cause (Woodward, 2003, p. 55):

14 See Woodward (2003, p. 98) for a technical definition. 
(DC): A necessary and sufficient condition for $X$ to be a direct cause of $Y$ with respect to some variable set $\langle\mathrm{V}>$ is that there be a possible intervention on $X$ that will change $Y$ when all other variables in $\langle\mathrm{V}\rangle$ are held fixed at some value by interventions.

Second, we also require the notion of a directed path or graph (Woodward, 2003, p. 42):

(P): A directed graph is an ordered pair $\langle V, E>$ where $V$ is a set of vertices that serve as the variables representing the relata of the causal relation and $E$ is a set of directed edges connecting these vertices. A directed edge from vertex or variable $X$ to vertex or variable $Y$ means that $X$ directly causes $Y \ldots$ A sequence of variables $\left\{V_{1}, \ldots V_{n}\right\}$ is a directed path from $V_{1}$ to $V_{n}$ if and only if for all $i(1 \leq i<n)$ there is a directed edge from $V_{i}$ to $V_{i}+1$.

Actual causation is defined in terms of a directed path, or "sequence" of direct causes. Relative to a causal model, $X=x$ is an actual cause of $Y=y$ iff:

(AC1): The actual value of $X=x$, and the actual value of $Y=y$.

(AC2): There is at least one directed path $\mathrm{P}$ from $X$ to $Y$ for which an intervention on $X$ will change the value of $Y$, given that other direct causes $Z_{i}$ of $Y$ that are not on this path have been fixed at their actual values.

Several items bear repeating. First, only values of variables can be actual causes. Second, only variables can be direct causes. Third, actual causation may hold between two variables' taking some values, even if direct causation does not. (For instance, consider three dominoes, represented by the variables $D_{1}, D_{2}$, and $D_{3}$, each of which takes the value 0 if the domino remains standing, and 1 if it topples. Now $D_{1}=1$ will be an actual cause of $D_{3}=1$, even though $D_{1}$ is not a direct cause of $D_{3}$.)

Finally, Hitchcock (2001, p. 287) places three criteria on a model's being appropriate:

(1) The equations must entail no false counterfactuals.

(2) The equations must not represent counterfactual dependence relations between events that are not distinct.

(3) The equations should not contain any variables whose values correspond to possibilities we consider too remote.

For Hitchcock, the first two criteria are objective, while the third is pragmatic. Woodward (2003, pp. 86-91) also endorses these criteria, placing particular importance on the third. Consider now a Woodward-style, causal-modelling treatment of a late-preemption case, "Billy and Suzy":

\section{Variables}

$B T=1$ if Billy throws; $=0$ if Billy does not throw

$S T=1$ if Suzy throws; $=0$ if Suzy does not throw

$B H=1$ if Billy's rock hits the bottle; $=0$ if Billy's rock does not hit the bottle 
$S H=1$ if Suzy's rock hits the bottle; = 0 if Suzy's rock does not hit the bottle $B S=1$ if bottle smashes; $=0$ if bottle does not smash

\section{Equations}

$B T=1$ or 0

$S T=1$ or 0

$B H=1$ if $(B T=1)$; else 0

$S H=1$ if $(S T=1$ and $B H=0)$; else 0

$B S=1$ if $(B H=1$ or $S H=1)$; else 0

Actual Case: $B T=1 ; S T=1 ; B H=1 ; S H=0 ; B S=1$

The corresponding graph is:

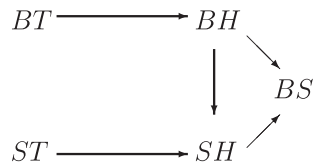

Here, $B T=1$ comes out as an actual cause of $B S=1$, which is intuitively correct. When we intervene on $B T=1$ and change its value to $B T=0$, while holding $S H=0$ fixed, we get a change in the value of $B S$ from $B S=1$ to $B S=0$. Thus, $B S=1$ is an actual cause of $B S=2$.

In words: the following ENF counterfactual is true: if Billy had not thrown his rock, but Suzy's rock had nevertheless not hit the bottle, then the bottle would not have smashed.

It may be objected that this "solution" is cheap. If we hold fixed that Suzy's throw does not hit the bottle, then the bottle's shattering counterfactually depends on Billy's throw. Yet the counterfactual of interest to us is what happens when Billy does not throw, all else remaining equal. In other words, although the ENF counterfactual is true, it is irrelevant to the problem of preemption. Simply because we can formulate such a counterfactual does not make it theoretically interesting. After all, if we are free to stipulate any further counterfactual interventions we want, then why not stipulate that the bottle does not shatter? That would also make the shattering dependent on Billy's throw. The ENF counterfactual on which the proposed solution depends is gerrymandered. So the solution is cheap. To cite Billy's throw as a cause, we need counterfactual dependence according to a nongerrymandered counterfactual. Nothing in the proposed solution speaks to that.

Note that this objection begs the question about which counterfactuals are of interest to a causal theory. Indeed, as Woodward (2003, p. 21) puts it:

I argue that the counterfactuals on which the philosophical tradition has tended to focus . . . are the wrong counterfactuals ... I argue that to elucidate certain kinds of causal claims, including ... singular causal claims, one must appeal to counterfactuals with complex antecedents counterfactuals that describe what would happen under combinations of manipulations or interventions, rather than under single manipulations. 
Hitchcock (2001, pp. 281-289) also takes his framework, which uses ENF counterfactuals, to provide a solution to early preemption, and there appears to be no reason why this treatment should not be extended to cover late preemption as well. And far from being gerrymandered specifically for the job of dealing with preemption, ENF counterfactuals constitute an integral component of the causal-modelling framework, which is itself independently motivated (see, e.g., Woodward, 2003, chs. 1, 2, 3).

Finally, neither Hitchcock nor Woodward claims that we are free to stipulate any further counterfactual interventions we want. For instance, condition (AC2) in Woodward's definition of actual (token) causation disallows us from stipulating that the bottle does not shatter, by way of an attempt to make the shattering dependent on Billy's throw.

At any rate, there is another problem. What does a variable's taking the value 0 represent? When we intervene on $B T$ in order to test whether $B T=1$ is an actual cause of $B S=1$, we hold $S H=0$ fixed. But doing so is ambiguous about what we are meant counterfactually to imagine occurring here: Suzy's rock just somehow does not hit the bottle (perhaps because she did not throw it). What event does $S H=0$ denote?

To put the problem another way, in Woodward's treatment of this case, $S H=0$ is ambiguous between:

(a) Suzy's rock does not hit the bottle, since she did not throw it (e.g., the rock stayed in her hand); and

(b) Suzy's rock does not hit the bottle because, although her rock passes right through the space where the bottle recently stood, Billy's rock hit the bottle a moment before.

So although Woodward's account appears to deal with late preemption, a problem about absences remains. This is because the variables are meant to represent or denote causal relata in the world. Moreover, although Woodward's account is implicitly contrastive, in that Billy's throwing rather than not throwing causes the bottle to smash rather than not to smash, the account is prevented from being explicitly contrastive since it remains unclear what it is that absence claims like $S H=0$ denote. So Woodward's account is inadequate. To see this more clearly, consider how Woodward might deal with the following claim:

My not watering my plant caused my plant not to blossom.

$\underline{\text { Variables }}$

$W=1$ if water plant; $=0$ if not

$D=1$ if plant blossoms; $=0$ if not 
Equations

$W=1$ or 0

$D=1$ if $(W=1)$; else 0

Actual Case: $W=0 ; D=0$.

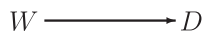

The difficulty in this case is that $W=0$ and $D=0$ each appear to denote nonactual events. So while we might appear to get the right answer, what we are implicitly saying about the world is open to a variant of the criticism that Schaffer's account apparently overcomes. Despite the fact that Woodward's account is implicitly contrastive, it is unclear in cases involving absences exactly what the contrast is meant to be.

\section{Proposal}

In this section, I propose a revised definition of causation - for the purposes of causal explanation - that handles absences and late preemption, by transposing Schaffer's account into a causal-modelling framework.

First,

- $\quad$ Let $\mathbf{C}$ be the set $\left\{x_{1}, x_{2}, \ldots x_{n}\right\}$ of all possible (non-zero) values for $X$, where $X$ is a variable in a model and $X$ 's taking any of its values would denote an actual, distinct event.

- $\quad$ Let $\mathbf{E}$ be the set $\left\{y_{1}, y_{2}, \ldots y_{n}\right\}$ of all possible (non-zero) values for $Y$, where $Y$ is a variable in a model and $Y$ 's taking any of its values would denote an actual, distinct event.

- Let $\mathbf{C}^{*}$ be the set $\mathbf{C} \backslash\left\{x_{1}\right\}$ of all possible alternative (non-zero) values to $x_{1}$, where $x_{1}$ denotes (by convention) the actual value of $X$, and where $X$ 's taking any of the alternative values $x_{i}$ of $\mathbf{C} \backslash\left\{x_{1}\right\}$ denotes a possible event alternative to $X=x_{1}$.

- $\quad$ Let $\mathbf{E}^{*}$ be the set $\mathbf{E} \backslash\left\{y_{1}\right\}$ of all possible alternative (non-zero) values to $y_{1}$, where $y_{1}$ denotes (by convention) the actual value of $Y$, and where $Y$ 's taking any of the alternative values $y_{i}$ of $\mathbf{E} \backslash\left\{y_{1}\right\}$ denotes a possible event alternative to $Y=y_{1}$.

Second, we define causation between variables in a model:

- $\quad \mathrm{X}$ is a direct cause of $Y$ in a causal model iff:

(i) $X$ and $Y$ are variables in that model;

(ii) For some assignment of values $\left\{v_{1}, \ldots v_{n}\right\}$ to all variables $V_{i}$ other than $X$ and $Y$ in the model, an intervention on $X$ changes the value of $Y$. 
In other words, $X$ is a direct cause of $Y$ iff the following counterfactuals $(\alpha)$ and $(\beta)$ are true:

For some $\left\{v_{1}, \ldots v_{n}\right\}$, there are $x_{r}, x_{s}, y_{r}, y_{s}\left(\right.$ with $\left.y_{r} \neq y_{s}\right)$ such that

( $\alpha) \quad O\left(X=x_{r}\right) \wedge O\left(V_{i}=v_{i}\right)>O\left(Y=y_{r}\right)$; and

( $\beta) \quad O\left(X=x_{s}\right) \wedge O\left(V_{i}=v_{i}\right)>O\left(Y=y_{s}\right)$.

Here, the operator " $>$ " represents the subjunctive conditional and is defined by what happens under interventions in a model. Hence, $(\alpha)$ and $(\beta)$ are explicitly non-foretracking (ENF) counterfactuals. Their truth is established as explained in Woodward's definition of a direct cause (see section 3 above). This definition is glossed above as conditions (i)-(ii).

In order to define actual causation, we must first recall Schaffer's contrastive definition of causation:

- $\quad c$ rather than $\mathbf{C}^{*}$ causes $e$ rather than $\mathbf{E}^{*}$ iff

i. $\quad c$ and $e$ are actual distinct events;

ii. $\quad \mathbf{C}^{*}$ is a set of possible event alternatives to $c$, and $\mathbf{E}^{*}$ is a set of possible event alternatives to $e$; and

iii. there is a one-one mapping from $\mathbf{C}^{*}$ to $\mathbf{E}^{*}$ by counterfactual entailment: $\left(\forall x \in \mathbf{C}^{*}\right)\left(\exists y \in \mathbf{E}^{*}\right)(O(x)>O(y)) \wedge\left(\forall y \in \mathbf{E}^{*}\right)\left(\exists x \in \mathbf{C}^{*}\right)(O(x)>O(y))$

Here, difference-making is a test for contrastive causation with singleton contrasts: $c$ rather than $c^{*}$ causes $e$ rather than $e^{*}$ iff $O\left(c^{*}\right)>O\left(e^{*}\right)$.

We import this definition into the causal-modelling framework:

- $X=x_{1}$ rather than $\mathbf{C}^{*}$ causes $Y=y_{1}$ rather than $\mathbf{E}^{*}$ iff:

(a) $X=x_{1}$ denotes an actual event where the actual (non-zero) value of $X=x_{1}$;

(b) $Y=y_{1}$ denotes an actual event, distinct from $X=x_{1}$, where the actual (non-zero) value of $Y=y_{1}$;

(c) $\mathbf{C}^{*}$ is the set $\left\{x_{2}, \ldots x_{n}\right\}$ of all possible alternative (non-zero) values to $x_{1}$, where $X$ 's taking any of these values $x_{i}$ denotes a possible event alternative to $X=x_{1}$;

(d) $\mathbf{E}^{*}$ is the set $\left\{y_{2}, \ldots y_{n}\right\}$ of all possible alternative (non-zero) values to $y_{1}$, where $Y$ 's taking any such values $y_{i}$ denotes a possible event alternative to $Y=y_{1}$;

(e) All direct causes $Z_{i}$ of $Y$ that are not on the path from $X$ to $Y$ are held fixed at their actual values $Z_{i}=z_{i}$ (i.e., there is a set of values $\left\{z_{1}, z_{2}, \ldots\right.$ $z_{n}$ \} for the $Z_{i}$ ); 
(f) An intervention on $X=x_{1}$ changes the value of $Y$; and

(g) There is a one-one mapping from $\mathbf{C}^{*}$ to $\mathbf{E}^{*}$ by ENF counterfactual entailment:

$$
\begin{aligned}
& \left(\forall x \in \mathbf{C}^{*}\right)\left(\exists y \in \mathbf{E}^{*}\right)\left(O ( X = x ) \wedge \left(O\left(Z_{i}=z_{i}\right)>(O(Y=y))\right.\right. \\
& \wedge\left(\forall y \in \mathbf{E}^{*}\right)\left(\exists x \in \mathbf{C}^{*}\right)\left(O(X=x) \wedge O\left(Z_{i}=z_{i}\right)>O(Y=y)\right)
\end{aligned}
$$

Thus, $X=x_{1}$ rather than $\mathbf{C}^{*}$ is a contrastive cause of $Y=y_{1}$ rather than $\mathbf{E}^{*}$ iff $(\gamma)$ is true:

$$
\begin{aligned}
& O\left(X=x_{2}\right) \wedge O\left(Z_{i}=z_{i}\right)>O\left(Y=y_{2}\right) \\
& \wedge O\left(X=x_{3}\right) \wedge O\left(Z_{i}=z_{i}\right)>O\left(Y=y_{3}\right) \\
& \wedge \ldots \\
& \wedge O\left(X=x_{n}\right) \wedge O\left(Z_{i}=z_{i}\right)>O\left(Y=y_{n}\right)
\end{aligned}
$$

The truth of $(\gamma)$ is analogous to the expanded version of Woodward's two necessary and jointly sufficient AC conditions on actual causation that I have listed above as conditions (a)-(g).

Next, we further restrict the contrastive relata to one alternative value each for $X$ and $Y$ :

- $X=x_{1}$ rather than $X=x_{i}$ causes $Y=y_{1}$ rather than $Y=y_{i}$ iff:

(h) $X=x_{1}$ denotes an actual event where the actual (non-zero) value of $X=x_{1}$;

(i) $Y=y_{1}$ denotes an actual event, distinct from $X=x_{1}$, where the actual (non-zero) value of $Y=y_{1}$;

(j) $X=x_{i}$ denotes a possible event alternative to $X=x_{1}$ where the (non-zero) value of $X=x_{i}$;

(k) $Y=y_{i}$ denotes a possible event alternative to $Y=y_{1}$ where the (non-zero) value of $Y=y_{i}$;

(1) All direct causes $Z_{i}$ of $Y$ that are not on the path from $X$ to $Y$ are held fixed at their actual values $Z_{i}=z_{i}$ (i.e., there is a set of values $\left\{z_{1}, z_{2}, \ldots z_{n}\right\}$ for the $\left.Z_{i}\right)$

(m) An intervention on $X=x_{1}$ changes the value of $Y: O(X=$ $\left.x_{i}\right) \wedge O\left(Z_{i}=z_{i}\right)>O\left(Y=y_{i}\right)$. 
(n) In the case of absence claims, the absence claim sets the relevant contrast $X=x_{i}$.

Thus, $X=x_{1}$ rather than $X=x_{i}$ is a selectively contrastive cause of $Y=y_{1}$ rather than $Y=y_{i}$ iff $(\delta)$ is true:

( $) \quad O\left(X=x_{i}\right) \wedge O\left(Z_{i}=z_{i}\right)>O\left(Y=y_{i}\right)$

The ENF counterfactual $(\delta)$, like $(\gamma)$, is analogous to the expanded version of Woodward's necessary and sufficient AC conditions on actual causation, here listed as conditions (h)-(n) above.

Finally, we will continue to bear in mind both Woodward's and Hitchcock's third criterion on a causal model's being appropriate, namely, that it should not contain any variables whose values correspond to possibilities that we consider too remote. Additionally, we will assume, along with Schaffer, that the contrast sets $\mathbf{C}^{*}$ and $\mathbf{E}^{*}$ are fixed by context.

\section{Solutions}

The transposed account is an improvement both on Schaffer's contrastive account and on Woodward's interventionist account. Regarding absence-causal claims, my account is an improvement on Woodward since it adequately deals with such claims. Regarding late preemption, my account is an improvement on Schaffer, since it successfully handles such cases.

\subsection{Absence Causation}

Consider the causal claim, "My not watering my plant caused my plant not to blossom."

As we saw before, Woodward's account is inadequate when it comes to absences. The account I have proposed revises Woodward's account so that variables may only take values denoting actual events. Moreover, we must explicitly delineate the possible contrastive alternatives. As an aid here, I adopt the convention of disallowing the value zero from causal models. Consider a revised model for the plant-watering case:

Variables

$W=1$ if water plant; $=2$ if nap

$D=1$ if plant blossoms; $=2$ if wilts

Equations

$W=1$ or 2

$D=1$ if $(W=1)$; else 2 
Actual Case: $W=2 ; D=2$.

$$
W \longrightarrow D
$$

The following ENF counterfactual is true with respect to this causal model: $O(W=1) \wedge O\left(Z_{i}=z_{i}\right)^{15}>O(D=1)$. From this ENF counterfactual, we get - relative to the causal model - the true contrastively causal claim:

$W=2$ rather than $W=1$ caused $D=2$ rather than $D=1$.

Or,

My napping rather than my watering my plant caused my plant to wilt rather than to blossom.

\subsection{Late Preemption}

By his own admission, Schaffer's account cannot deal with late preemption, despite its success in dealing with absences. Conversely, a Woodward-style causalmodelling account successfully deals with late preemption, despite remaining problematic in terms of absences. I will now show how my transposed account both (i) handles late preemption and (ii) does so in a way that adequately deals with absences. This not only remedies Woodward's account by adequately dealing with absences, but it remedies Schaffer's account in being able to handle preemption.

Assumption: throwing a rock always hits the bottle (if it is there); lobbing it always misses.

\section{Variables}

$B T=1$ if Billy throws his rock; $=2$ if Billy lobs his rock

$S T=1$ if Suzy throws her rock; =2 if Suzy lobs her rock

$B H=1$ if bottle is hit by Billy's rock (at $\left.t_{1}\right) ;=2$ if bottle continues to stand (at $t_{1}$ )

$S H=1$ if Suzy's rock hits bottle (at $\left.t_{2}\right) ;=2$ if Suzy's rock hits tree (at $t_{2}$ )

$B S=1$ if bottle smashes; $=2$ if bottle remains intact

\section{Equations}

$B T=1$ or 2

$S T=1$ or 2

$B H=1$ if $(B T=1)$; else 2

$S H=1$ if $(S T=1$ and $B H=2)$; else 2

$B S=1$ if $(B H=1$ or $S H=1)$; else 2

15 Note that the second conjunct of the antecedent of this counterfactual is not needed here (as it happens). 
We have the usual graph in this case:

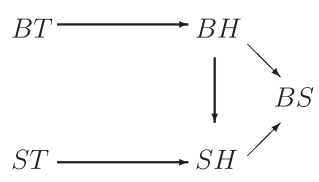

Here, $B T=1$ comes out as an actual (selective) contrastive cause of $B S=1$, since if we intervene on $B T=1$ and change it to $B T=2$, while holding $S H=2$ fixed, the value of $B S$ changes. (Essentially, this is the same solution as before, only modified for explicit contrastivity.) Put another way, the following ENF counterfactual is true with respect to this model:

$O(B T=2) \wedge O(S H=2)>O(B S=2)$

Although this counterfactual does not correspond to any single structural equation, it is still made true in virtue of them, together with my expanded AC-like conditions. Additionally, a particular (and true) contrastively causal claim follows from this ENF counterfactual. That is,

$B T=1$ rather than $B T=2$ causes $B S=1$ rather than $B S=2$.

Or,

Billy's throwing his rock rather than lobbing it actually causes the bottle to smash rather than to remain intact.

Of course, this causal-explanatory judgement is made true only in virtue of the ENF counterfactual stated above, since unless we hold fixed Suzy's rock hitting the tree, we must conclude that even if Billy lobbed his rock, and thus the bottle remained standing rather than being hit by Billy's rock, Suzy's rock would have hit and smashed the bottle instead.

\section{Conclusion}

Adequately handling the way in which absences are cited as relata in many of our explanations of why things happen presents grave difficulties for any account of causal explanation. Schaffer's quaternary, contrastive account resolves these difficulties. Yet Schaffer's account cannot handle preemption cases, which also present a difficulty for most accounts of causation, or of causal explanation. Woodward's account handles late preemption, by using ENF counterfactuals. Yet his account is inadequate when it comes to absences. In order to overcome these shortcomings, I have proposed a definition of actual causation - for the purposes of causal 
explanation - that handles both absences and late preemption. I arrive at this definition by transposing Schaffer's account into an interventionist, causalmodelling framework.

Of course, one might say of my amendment to Woodward's account, just as one might say of Woodward's causal-modelling strategy itself, that the view already exists, and in a simpler form. On Stephen Yablo's (2002) view, the way in which effects depend on their causes requires our holding fixed causal conditions, in such $a$ way that in a case of preemption we are essentially holding fixed that Suzy's rock does not hit the bottle, i.e., when we assess whether Billy's throwing his rock caused the bottle to smash. This is what Yablo calls (2002, p. 138) "de facto dependence" of the bottle's smashing on Billy's throw. Be this as it may, it is not obvious that Yablo's account is to be preferred, since it is not clear whether it adequately handles absences. By contrast, both Schaffer's and my accounts do handle absences. The causal-modelling framework also has a significant advantage over Yablo's view as a theory of causal explanation, since it models how scientific investigation actually identifies causes in experimental settings.

On the other hand, one might think that precisely this reliance on the causalmodelling framework is problematic. For instance, Ned Hall (2007, p. 110) thinks that the central problem facing modelling approaches is their failure to make clear what is going on in the "distinction between the default behavior of an object or system, and deviations therefrom," where the default behaviour is the behaviour it would exhibit, if nothing acted on it. Yet it is precisely this issue that I have tried to address here, by building in Schaffer's explicit contrastivity into a Woodward-style modelling approach.

\section{Acknowledgements}

Thanks to Paul Bartha, Roberta Ballarin, Andrew Irvine, Carolina Sartorio, and an anonymous referee at this journal for helpful suggestions and comments on earlier drafts of this article.

\section{References}

HaLl, N. (2004) “Two Concepts of Causation.” In J. Collins, N. Hall and L. A. Paul (eds), Causation and Counterfactuals, pp. 225-276. Cambridge, MA: MIT Press.

HaLl, N. (2007) "Structural Equations and Causation." Philosophical Studies 132: 109-136. HiтCHCOCK, C. R. (2001) “The Intransitivity of Causation Revealed in Equations and Graphs." Journal of Philosophy 98: 273-299.

LEWIS, D. (2000) “Causation as Influence.” Journal of Philosophy 97: 182-197. 
Livengood, J. and Machery, E. (2007) “The Folk Probably Don't Think What You Think They Think: Experiments on Causation by Absence." Midwest Studies in Philosophy 31: $107-127$.

Lombrozo, T. (2010) "Causal-Explanatory Pluralism: How Intentions, Functions, and Mechanisms Influence Causal Ascriptions.” Cognitive Psychology 61: 303-332.

MACKIE, J. L. (1974) The Cement of the Universe: A Study of Causation. Oxford: Clarendon Press.

SCHAfFer, J. (2005) “Contrastive Causation.” Philosophical Review 114: 327-358.

Woodward, J. (2003) Making Things Happen: A Theory of Causal Explanation. New York: Oxford University Press.

YABLO, S. (2002) “De Facto Dependence.” Journal of Philosophy 99: 130-148. 